\title{
Calidad del seguimiento a la exposición perinatal al HIV y observancia de las estrategias reconocidas para disminuir su transmisión en un centro de referencia de Medellín
}

Catalina Arango-Ferreira',2, Diana Isabel Villegas², Laura Daniela Burbano², Augusto Quevedo ${ }^{1,2}$

${ }^{1}$ Hospital San Vicente Fundación, Medellín, Colombia

${ }_{2}^{2}$ Departamento de Pediatría, Universidad de Antioquia, Medellín, Colombia

Introducción. La prevención de la transmisión materno-infantil del virus de la inmunodeficiencia humana (Human Immunodeficiency Virus, HIV) es una estrategia fundamental para evitar la infección en niños. A nivel local, se desconoce la situación de las mujeres gestantes infectadas por HIV y el grado de observancia de las estrategias reconocidas mundialmente para disminuir la transmisión perinatal.

Objetivo. Describir las características sociodemográficas y clínicas de las mujeres gestantes con HIV y de sus hijos en un centro de referencia de Medellín.

Materiales y métodos. Se hizo un estudio descriptivo retrospectivo entre 2012 y 2015 mediante la revisión de las historias clínicas de las mujeres gestantes con HIV y de sus neonatos habidos en partos atendidos en el Hospital San Vicente Fundación. Se describieron las variables de atención prenatal, parto, recién nacido y seguimiento de los neonatos. Resultados. Se analizaron 106 madres y sus hijos expuestos al HIV. El 39,6\% de las mujeres gestantes conocía el diagnóstico antes del embarazo y al 58,5\% se le diagnosticó durante este. El 95,3\% de las mujeres gestantes asistió a control prenatal, 46,5\% de ellas a partir del primer trimestre. Si bien el $95 \%$ recibió antirretrovirales, el 23,9 \% comenzó a tomarlos tardíamente en el tercer trimestre. Solo el $63 \%$ de las mujeres registró carga viral para el HIV después de la semana 34 de gestación. El 90,6 \% de los partos fueron por cesárea, y el virus del papiloma humano y la sífilis fueron las principales infecciones concomitantes. Se hizo seguimiento de 103 niños (no fue posible localizar a tres de ellos para el seguimiento), a ninguno se le confirmó la presencia del HIV y, en el $88 \%$, se descartó.

Conclusiones. En este estudio, no se registraron casos de transmisión perinatal. Sin embargo, siguen presentándose fallas y retrasos en la atención prenatal y en la oportunidad del seguimiento materno para confirmar oportunamente el HIV y para detectar tempranamente infecciones maternas concomitantes que eviten la morbilidad y las secuelas en los neonatos.

Palabras clave: HIV; recién nacido; transmisión de enfermedad infecciosa; atención perinatal; estrategias mundiales.

Follow up of HIV perinatal exposure and accomplishment of strategies to reduce the risk of viral transmission, experience in a reference hospital in Medellín

Introduction: Prevention of mother-to-child transmission of the human immunodeficiency virus (HIV) is essential to limit the spread of the disease. Colombian data about HIV infection in pregnancy are scarce, as well as on the results of the strategies used worldwide to reduce perinatal transmission.

Objective: To describe the characteristics and outcomes of pregnant women infected with HIV and their children in a reference center in Medellín.

Materials and methods: We conducted a retrospective observational study for the 20122015 period by studying the clinical records of newborns exposed to HIV and their mothers. We evaluated the characteristics of prenatal care, deliveries, and infant postnatal care, as well as the follow-up data to confirm or exclude HIV transmission.

Results: We included 106 infants and their mothers. We found that $39,6 \%$ of mothers knew about the HIV diagnosis before pregnancy and $58,5 \%$ were diagnosed during pregnancy; $95.3 \%$ of them attended prenatal controls, but only $46.5 \%$ as of the first trimester; $95 \%$ of them received antiretrovirals, but $23.9 \%$ started therapy just during the third trimester. Only $63 \%$ of women had a viral load for HIV after 34 weeks of gestation. None of the 103 children with follow up had confirmed presence for HIV and in $88 \%$ of them, it was discarded.

Conclusions: No cases of perinatal HIV transmission were found in the study. However, difficulties and delays persist in prenatal care, in timely maternal follow-up to confirm or discard HIV, and for early detection of maternal co-infections and their effects on newborns.

Keywords: HIV; infant, newborn; disease transmission, infectious; perinatal care; world strategies. 
La infección por el virus de la inmunodeficiencia humana (Human Immunodeficiency Virus, HIV) es reconocida como una pandemia por su crecimiento exponencial. Esto llevó a la necesidad de desarrollar e implementar estrategias para impedir su transmisión, con lo cual se logró disminuir en $70 \%$ el número de nuevas infecciones en el 2015 comparado con el 2000 , pues pasaron de 490.000 casos a 150. En el 2004 se reportaron 1,8 millones de niños infectados por el HIV en el mundo, es decir, el $4 \%$ de la población afectada (1), en tanto que, en Colombia, se reportaron entre 44.000 y 65.000 personas huérfanas a causa del HIV (2). El $90 \%$ de los niños con HIV adquieren la infección por transmisión perinatal, por lo cual la prevención de la transmisión materno-infantil es un pilar fundamental para evitar la propagación del HIV y las consecuencias clínicas y sociales que este diagnóstico conlleva para los niños (1).

En el marco de la prevención de la transmisión materno-infantil del HIV, se han establecido medidas para lograr el diagnóstico y el inicio temprano del tratamiento en las mujeres gestantes; entre ellas cabe mencionar el ingreso oportuno al programa de control prenatal, la calidad adecuada de dicho programa, la tamización para HIV durante el primer trimestre de la gestación y el acceso inmediato a una segunda prueba para la confirmación diagnóstica en caso de que la primera resulte reactiva. Además, existen otras estrategias específicas que buscan minimizar la carga viral a la cual pueda verse expuesto el feto durante la gestación y en el momento del parto, así como evitar la exposición posnatal del recién nacido al virus.

Tales estrategias incluyen el inicio temprano de tratamiento antirretroviral, los controles prenatales periódicos para verificar su cumplimiento y las pruebas de laboratorio para verificar la reducción de la carga viral, así como la infusión intravenosa de zidovudina durante el parto en aquellos casos en que esta sea mayor de 1.000 copias $/ \mathrm{ml}$, idealmente en una fecha cercana al parto, entre las semanas 34 y 36 . La cuantificación de la carga viral justo antes del parto también ayuda a decidir la vía del nacimiento, es decir, cesárea cuando hay más de $1.000 \mathrm{copias} / \mathrm{ml}$. Una vez nacido el bebé, se recomienda administrarle antivirales como profilaxis y, según las normas del país, suprimir la lactancia materna. El cumplimiento estricto de todas estas acciones ha dado como resultado la reducción del riesgo de transmisión de $45 \%$ hasta $102 \%$ (3).

En Latinoamérica la reducción de las infecciones perinatales varía según los países (1). Un ejemplo regional de la importancia de cumplir estrictamente con estas estrategias preventivas contra la transmisión materno-infantil del HIV es Cuba, país que, con el aval de la OMS, reportó recientemente la eliminación de esta vía de transmisión del HIV y de la sífilis.

En el 2012, se reportaron en Colombia 781 mujeres gestantes con HIV (4) y se estima que el diagnóstico de HIV por transmisión materno-infantil se estableció en 112 niños en el 2012 y en 108 en el 2013, con una tasa de $4,6 \%$ de transmisión vertical del virus, frente a una meta de menos del $2 \%$ para el país (5). A nivel local, no se sabe cuál es la situación actual de las mujeres gestantes con infección por HIV en cuanto a los factores conocidos que previenen la transmisión materno-infantil y el diagnóstico de HIV de sus recién nacidos, razón por la cual se planteó el presente estudio.

\section{Materiales y métodos}

Se trató de un estudio descriptivo retrospectivo basado en la revisión de las historias clínicas de los recién nacidos de mujeres con infección por HIV diagnosticada antes del embarazo, durante la gestación o inmediatamente 
después del parto y registrados bajo el código CIE-10: Z206 (contacto con el virus de la inmunodeficiencia humana y exposición a él). Esta información se cruzó con la base de datos del registro de interconsultas atendidas por el Servicio de Infectología Pediátrica del Hospital Universitario San Vicente Fundación de Medellín.

El estudio se llevó a cabo entre el $1^{\circ}$ de octubre de 2012 y el 30 de septiembre de 2015. Se excluyeron las historias clínicas de las madres cuyo parto fue atendido por fuera de esta institución. Por protocolo institucional, todos los niños con exposición perinatal a HIV son evaluados por profesionales en infectología pediátrica, por lo que el total de niños reportados en los registros médicos se comparó con el registro de interconsultas de infectología pediátrica del hospital. A partir de la historia clínica de los neonatos, se accedió a la historia materna para obtener los datos del control prenatal y los relacionados con la atención del parto y el manejo inicial del recién nacido.

Para determinar el diagnóstico de infección por HIV en el recién nacido, se obtuvieron los datos del registro de seguimiento de mujeres gestantes con diagnóstico de HIV y de menores expuestos de la Secretaría Seccional de Salud y Protección Social de Antioquia del 2012 al 2016.

Como caso de diagnóstico descartado en los lactantes, se consideró el hijo de una madre con infección por HIV y resultados negativos en la evaluación de carga viral y que una evaluación se hubiera obtenido a los cuatro meses de vida, o en una edad posterior, y como diagnóstico confirmado, el tener por lo menos una carga viral positiva confirmada por una segunda prueba.

Se extrajeron de la historia clínica los datos de las variables sociodemográficas y las propias del control prenatal, la atención del parto, el recién nacido y el seguimiento hasta el año de edad. Se diligenció un formato que incluía las variables de análisis y que fue previamente probado mediante un estudio piloto con cinco historias clínicas para determinar aquellas variables que requirieran ajustes, las cuales fueron analizadas por todos los investigadores para determinar los cambios pertinentes.

Para el análisis estadístico, se ingresaron las variables en un formulario de recolección electrónico en Microsoft Excel y se analizaron después con el programa IBM SPSS ${ }^{\mathrm{TM}}$; se cruzaron y se calcularon las frecuencias y los porcentajes.

El estudio fue aprobado por los comités de ética del Hospital Universitario San Vicente Fundación y de la Universidad de Antioquia. Se mantuvo la confidencialidad de la información de cada una de las pacientes y de los neonatos evaluados, y esta no se reveló ni discutió con los evaluadores ni con su entidad prestadora de servicios de salud.

\section{Resultados}

Durante el periodo de observación, se encontraron 122 historias clínicas de madres y sus hijos, de las cuales se excluyeron 16 porque el parto no se atendió en el hospital de estudio, por lo que, finalmente, se analizó la información de 106 recién nacidos de madres con diagnóstico de infección por HIV. La edad promedio de las madres fue de 23,5 años y $55,7 \%$ de ellas provenía de fuera de Medellín. Tres de ellas eran habitantes de calle y nueve consumían sustancias psicoactivas; de estas últimas, tres no lo hacían por vía intravenosa y seis consumían sustancias no especificadas. 
La mayoría de las pacientes asistió a los controles prenatales $(95,3 \%)$ y se diagnosticó la infección por HIV en el 58,5 \% de los casos. El 39,6\% de las mujeres gestantes conocía su diagnóstico de HIV antes de la gestación, razón por la cual habían sido incluidas en el estudio. En las gestantes diagnosticadas durante el embarazo, el diagnóstico se hizo en una edad gestacional promedio de 19 semanas. En el $20 \%$ de las madres, la infección se diagnóstico durante el primer trimestre y, en el $14,5 \%$, a partir de las 32 semanas de gestación. En promedio, se hicieron cinco controles prenatales por cada mujer gestante.

El $95 \%$ de las madres infectadas con HIV que tuvieron control prenatal recibieron tratamiento antirretroviral y, en 39,5\% de ellas, se inició en el segundo trimestre de gestación (cuadro 1).

Se encontraron 19 pacientes con carga viral de 1.000 o más copias $/ \mathrm{ml}$, de las cuales solo nueve registraron carga viral después de la semana $34(8,4 \%)$. Estas nueve pacientes recibían tratamiento antirretroviral iniciado después del primer trimestre y, en seis de ellas, iniciado en el último trimestre. Una paciente presentó intolerancia por vómito, otra tuvo acceso irregular a los medicamentos y no se conoció la fecha de inicio del tratamiento antirretroviral en otra. De las 52 mujeres gestantes con una carga viral de menos de 1.000 copias/ml después de la semana 34, 50 recibían el tratamiento y 40 de ellas lo habían iniciado antes del último trimestre.

En 84 pacientes el parto fue a término y, en 53 (63\%) de estas se detectó carga viral después de las 34 semanas; 19 mujeres gestantes tenían una carga viral de 1.000 o más copias/ml y, de ellas, 18 recibían tratamiento. Según la genotipificación, ninguna de ellas presentaba mutaciones resistentes al tratamiento antirretroviral. En el 18,4\% de los casos se reportó un conteo de linfocitos T CD4+ (LTCD4) menor de 200.

El parto fue por cesárea en la mayoría $(90,6 \%$ ) de los casos y, en el 91,5\% de los partos se administró zidovudina intravenosa (AZT). A nueve (8,5\%) pacientes no se les administró zidovudina intravenosa durante el parto: tres que ingresaron en trabajo de parto, dos con diagnóstico después del parto, una por decisión de ginecología, una que no la aceptó, una que llegó al parto sin conocerse el diagnóstico y una última en cuya historia clínica no se registró la razón.

El porcentaje de partos prematuros fue de $20 \%$, el de niños con bajo peso al nacer (menos de $2.500 \mathrm{~g}$ ), de 24,5\%, y el de aquellos con retardo del crecimiento intrauterino, de $11 \%$. A todos los recién nacidos se les inició profilaxis antirretroviral y a uno de ellos no se le restringió la lactancia materna. Entre las demás medidas descritas, las más frecuentemente usadas fueron el baño del recién nacido y el lavado gástrico (cuadro 2).

De los 106 niños estudiados, se hizo el seguimiento de 103 (97\%), dos de ellos por estar afiliados a la seguridad social por fuera del departamento de Antioquia. Ninguno de los niños incluidos en el estudio tuvo diagnóstico confirmado de HIV.

El diagnóstico se descartó en 93 de las 106 pacientes evaluadas; se hizo segumiento posnatal a 103 niños. La infección se descartó mediante dos cargas virales negativas, una al mes y otra a los cuatro meses de vida, en 97 niños. En el resto de los niños, las cargas virales se determinaron en tiempos más tardíos (cuadro 3). 
Cuadro 1. Características maternas

\begin{tabular}{|c|c|c|}
\hline \multicolumn{3}{|l|}{ Características } \\
\hline Edad materna (años)* & \multicolumn{2}{|c|}{$23,5(15-40)$} \\
\hline Hijos vivos $(n)^{*}$ & 2 & $(1-6)$ \\
\hline \multicolumn{3}{|l|}{ Procedencia [n (\%)] } \\
\hline Medellín & 47 & $(44,3)$ \\
\hline Afueras de Medellín & 59 & $(55,7)$ \\
\hline \multicolumn{3}{|l|}{ Residencia [n (\%)] } \\
\hline Urbana & 89 & $(83,9)$ \\
\hline Rural & 17 & $(16,1)$ \\
\hline \multicolumn{3}{|l|}{ Estado civil [n (\%)] } \\
\hline Unión libre & 56 & (53) \\
\hline Soltera & 41 & $(38,6)$ \\
\hline Otros $^{\dagger}$ & 9 & $(8,4)$ \\
\hline \multicolumn{3}{|l|}{ Diagnóstico de la pareja [n (\%)] } \\
\hline Positivo & 23 & $(21,7)$ \\
\hline Negativo & 14 & $(13,2)$ \\
\hline Desconocido & 31 & $(29,3)$ \\
\hline Sin datos & 38 & $(35,8)$ \\
\hline \multicolumn{3}{|l|}{ Seguridad social [n (\%)] } \\
\hline Subsidiado & 95 & $(89,6)$ \\
\hline Contributivo & 10 & $(9,5)$ \\
\hline Sin afiliación & 1 & $(0,9)$ \\
\hline \multicolumn{3}{|l|}{ Otras ETS [n (\%)] } \\
\hline No & 82 & $(77,4)$ \\
\hline Sí & 24 & $(22,6)$ \\
\hline Condilomatosis & 11 & $(45,8)$ \\
\hline Sífilis & 8 & $(33,3)$ \\
\hline Hepatitis B & 2 & $(8,4)$ \\
\hline Otras $^{\ddagger}$ & 3 & $(12,5)$ \\
\hline Asistencia a control prenatal [n (\%)] & 101 & $(95,3)$ \\
\hline Controles prenatales $(n)^{* s}$ & 5 & $(1-14)$ \\
\hline \multicolumn{3}{|l|}{ Inicio del control prenatal [n (\%)] } \\
\hline Primer trimestre & 47 & $(46,5)$ \\
\hline Segundo trimestre & 27 & $(26,7)$ \\
\hline Tercer trimestre & 10 & $(9,9)$ \\
\hline Sin datos & 17 & $(16,9)$ \\
\hline Primera prueba de tamización (semanas)* & 17 & $(6-35)$ \\
\hline \multicolumn{3}{|l|}{ Momento del diagnóstico } \\
\hline Antes de la concepción & 42 & $(39,6)$ \\
\hline Prenatal & 62 & $(58,5)$ \\
\hline Después del parto & 2 & $(1,9)$ \\
\hline Momento del diagnóstico prenatal (semanas) & 19 & $(6-37)$ \\
\hline \multicolumn{3}{|l|}{ Seguimiento $[\mathrm{n}(\%)]^{* *}$} \\
\hline Ginecología & 90 & $(84,9)$ \\
\hline Infectología & 32 & $(30,2)$ \\
\hline Tratamiento [n (\%)] & 96 & $(90,6)$ \\
\hline \multicolumn{3}{|l|}{ Momento de inicio del tratamiento [n (\%)] } \\
\hline Antes de la concepción & 15 & $(15,7)$ \\
\hline Primer trimestre & 18 & $(18,7)$ \\
\hline Segundo trimestre & 38 & $(39,8)$ \\
\hline Tercer trimestre & 23 & $(23,9)$ \\
\hline Sin datos & 2 & $(1,9)$ \\
\hline Evaluación de carga viral prenatal [n (\%)] & 91 & $(85,8)$ \\
\hline EG de la última carga viral, semanas * & 32 , & (14-39) \\
\hline \multicolumn{3}{|l|}{ Valor de la última carga viral a [número de copias/ml (\%)] } \\
\hline$<40$ & 38 & $(41,7)$ \\
\hline $41-1.000$ & 34 & $(37,5)$ \\
\hline$\geq 1.000$ & 19 & $(20,8)$ \\
\hline Disponibilidad de conteo de LT CD4 [n (\%)] [n (\%)] & 92 & $(86,8)$ \\
\hline \multicolumn{3}{|l|}{ Conteo de linfocitos T CD4 (células/ml) [n (\%)] } \\
\hline$\geq 200$ & 75 & $(81,5)$ \\
\hline$<200$ & 17 & $(18,4)$ \\
\hline
\end{tabular}

ETS: enfermedades de transmisión sexual; VPH: virus del papiloma; EG: edad de gestación * Promedio y rango

† Otros estados civiles: casada (1), viuda (1), desconocido (7)

‡ Otras ETS: tricomoniasis (2), hepatitis C (1)

$s$ Valores y porcentajes tomados de las mujeres gestantes que asistieron a control prenatal

a Carga viral indetectable: $<40$ copias $/ \mathrm{ml}$ 
Se desconocía el diagnóstico en 13 (12\%) de los 106 pacientes, dos de los cuales pesaron menos de $2.500 \mathrm{~g}$ al nacer y fallecieron por causa desconocida, aunque recibieron tratamiento y se tomaron las medidas necesarias para disminuir la transmisión viral durante el parto. En estos dos niños, el diagnóstico materno prenatal se hizo después del segundo trimestre de gestación y la primera carga viral (determinada durante el primer mes de nacidos) fue negativa. Uno, cuya madre consumía sustancias psicoactivas, presentó depresión neonatal y malformaciones de las vías genitales, urinarias y gastrointestinales, y el otro era un niño indígena.

Cuadro 2. Características del parto y del recién nacido

\begin{tabular}{lr}
\hline Madres & $\mathbf{n}(\%)$ \\
\hline Edad gestacional en el momento del parto (semanas) & \\
$\quad<37$ & $22(20,8)$ \\
$\quad \geq 37$ & $84(79,2)$ \\
Tipo de parto & \\
$\quad$ Cesárea & $96(90,6)$ \\
$\quad$ Vaginal & $10(9,4)$ \\
Ruptura de membranas antes del parto (horas) & \\
$\quad \geq 4$ & $8(7,5)$ \\
$\quad<4$ & $7(87,5)$ \\
Inicio de AZT intravenosa & $97(91,5)$ \\
\hline Recién nacidos & \\
\hline Sexo masculino & $53(49,1)$ \\
Peso (g) & \\
$\quad \geq 2.500$ & $80(75,5)$ \\
$<2.500$ & $26(24,5)$ \\
RCIU & $12(11,3)$ \\
Inicio de profilaxis <12 horas & $96(90,6)$ \\
Baño del recién nacido & $98(92,5)$ \\
Lavado gástrico & $26(24,5)$ \\
Restricción de lactancia materna & $105(99,1)$ \\
\hline AZT:
\end{tabular}

AZT: zidovudina; RCIU: retardo del crecimiento intrauterino

Cuadro 3. Seguimiento de los recién nacidos

\begin{tabular}{lr}
\hline Seguimiento & $\mathbf{n}(\%)$ \\
\hline Primera carga viral (meses) & $103(97)$ \\
$\leq 1$ & $86(83,5)$ \\
$1-4$ & $14(13,5)$ \\
$\geq 5$ & $3 \quad(3)$ \\
Segunda carga viral (meses) & $97(94,2)$ \\
$<4$ & $4(4,1)$ \\
A los 4 & $54(55,7)$ \\
Entre los 5 y los 12 & $34(35)$ \\
$>12$ & $5(5,2)$ \\
Diagnóstico HIV & $103 \quad(97)$ \\
Confirmado & \\
Descartado & $94(88,7)$ \\
Desconocido & \\
\hline
\end{tabular}

† Se comprobó la segunda carga viral antes de los 4 meses de vida: 4 niños; sin seguimiento: 3 niños; no se comprobó la segunda carga viral: 3 niños; fallecidos: 2 niños

\section{Discusión}

En el 2013, el porcentaje de transmisión vertical de HIV en Antioquia se estimaba en $2,1 \%$ (6). Sin embargo, no se ha reportado el grado de 
cumplimiento de medidas como el diagnóstico oportuno, el tratamiento y otras estipuladas para reducir este porcentaje, por lo que es importante que el personal de salud sea consciente de dichos factores y de cómo se comportan actualmente.

En este estudio, más de la mitad de las mujeres gestantes con diagnóstico de HIV procedía de fuera de Medellín, quizá porque el Hospital Universitario San Vicente Fundación es un centro de referencia departamental. La mayoría de ellas había entrado en la tercera década de la vida, cuando la tendencia global del diagnóstico de novo de la infección por HIV es a aumentar en mujeres más jóvenes (7). En este estudio, el porcentaje de mujeres gestantes menores de 20 años $(22,6 \%)$ con diagnóstico de HIV fue mayor comparado con los datos de hace de diez años en Colombia (17\%) (8), así como a nivel de otras poblaciones del país (13,2\%).

En un estudio del 2009 sobre neurodesarrollo de hijos de madres expuestas al HIV en la misma institución, la edad materna promedio fue de 26 años, ligeramente mayor a la de la población del presente estudio (9). Otras diferencias sociodemográficas incluyeron el elevado porcentaje de mujeres solteras (39\%) y una mayor cobertura de seguridad social, en comparación con los datos reportados en Bogotá en el 2014 (10). Se reportó cerca de $22 \%$ de madres cuyas parejas tenían infección por HIV, pero en el $65 \%$ de los casos se desconocía el estado serológico de la pareja, principalmente, porque este dato no se registró en la historia clínica. Además, en el $35 \%$ de estas madres se desconocía el diagnóstico de infección por HIV en otros de sus hijos.

No se encontraron estudios previos que reportaran estos datos pero, si el objetivo global es disminuir la transmisión del HIV (11) y garantizar la detección temprana de los niños infectados, es necesario indagar sobre esto durante los controles prenatales y en la atención perinatal. Casi una cuarta parte de las madres incluidas tenía otras infecciones de transmisión sexual, porcentaje este similar al reportado en Chile (20\%). En el presente estudio, predominaron la condilomatosis (33\%) y la sífilis $(8,4 \%)$, en tanto que la sífilis ocupó el primer lugar en Chile (12). Actualmente, estas condiciones pueden prevenirse mediante la vacunación (condilomatosis) o tratarse (sífilis), pero cuando no se detectan tempranamente para administrar el tratamiento, implican una mayor morbilidad para el recién nacido.

Cerca del $40 \%$ de las madres del presente estudio habían sido diagnosticadas antes de la concepción, lo que es similar a lo reportado en Estados Unidos en niños con infección adquirida por transmisión maternoinfantil confirmada (41\%) (13); en Chile, este porcentaje no sobrepasa el 10 $\%$ y a nivel nacional fue del $16 \%$ en el $2010(14,15)$.

También, debe señalarse que menos del $50 \%$ de las pacientes con diagnóstico previo a la concepción empezó a asistir al control prenatal en el primer trimestre. Frente a esta situación, a nivel mundial se han planteado estrategias como la llamada $\mathrm{B}+$, la cual propone continuar el tratamiento antirretroviral de por vida en mujeres embarazadas con HIV, independientemente del conteo de LT CD4, para así reducir la transmisión del HIV a sus hijos y parejas (16). Este tipo de estrategia solucionaría parcialmente el problema encontrado en este estudio de un porcentaje alto de niños con hermanos cuyo estado de infección se desconocía, al igual que el del padre, así como el del inicio tardío del tratamiento en las mujeres con diagnóstico 
previo. Estos resultados reiteran la importancia de implementar la estrategia $\mathrm{B}+$ en las guías colombianas para la prevención de la transmisión materno-infantil en embarazos futuros, aunque son necesarios estudios adicionales.

Comparados con los datos reportados en el país, la asistencia al control prenatal registrada en este estudio es la mejor en mujeres gestantes positivas para el HIV, aunque hay que señalar que menos de la mitad comenzó a asistir a dicho control oportunamente (en el primer trimestre), por lo que la primera prueba de tamización para HIV fue tardía, en promedio, en la semana 17. Este aspecto influye en la demora de casi tres semanas para confirmar el diagnóstico. Asimismo, el diagnóstico tardío (después de la semana 32 de gestación) se siguió presentando en el 17,1 \% de la población, aunque en menor medida que en otras regiones del país, donde esta cifra alcanza el 47,5\% (10).

Este dato respaldaría la decisión de hacer un estudio para HIV durante el tercer trimestre del embarazo en todas las mujeres gestantes y no solo en aquellas en quienes se hayan detectado 'conductas de riesgo'. El diagnóstico después del parto se presentó en una paciente con resultado negativo en la primera de ELISA y cuya pareja tenía diagnóstico de HIV, lo que pondría de presente la pertinencia de repetir la prueba de tamización en el tercer trimestre cuando se presenten conductas de riesgo (17).

Se reportó un bajo acceso a la consulta prenatal con infectología en el grupo de mujeres gestantes estudiadas y, por el contrario, a casi todas las que tuvieron control prenatal se les hizo seguimiento de ginecología. Por ello, se sugiere que los ginecólogos sean los profesionales de salud que lideren la atención prenatal y perinatal de las madres con infección o exposición al HIV y la de sus bebés.

Se registró un acceso adecuado al tratamiento antirretroviral si se tiene en cuenta que la meta mundial es del $90 \%$ de mujeres gestantes con tratamiento; el porcentaje en este estudio fue similar a las cifras reportadas en Europa y mayor que el reportado a nivel global y en Latinoamérica (77 y $88 \%$, respectivamente) (18), así como al porcentaje nacional en el 2010 (14). En el $40 \%$ de las mujeres gestantes se encontró que el tratamiento antirretroviral se inició en el segundo trimestre, lo que se explica porque en este periodo se hace la mayoría de los diagnósticos prenatales, pero no puede considerárselo tardío, ya que en las guías nacionales anteriores al 2014 se indicaba iniciar el tratamiento después de la semana 14 de gestación, es decir, en el segundo trimestre. Sin embargo, todavía hay un porcentaje importante $(23,9 \%)$ de pacientes que inicia el tratamiento en el tercer trimestre, por lo que debe mejorarse su oportunidad y reforzar en este grupo todas las medidas para evitar la transmisión materno-infantil.

En diversas guías de atención se recomienda determinar la carga viral entre las semanas 34 y 36 de gestación $(11,19)$, lo cual es de especial importancia para definir la vía del parto y reducir, así, la exposición al virus en el canal del parto en los casos de mayor riesgo, y determinar la profilaxis necesaria para el recién nacido sin incurrir en el uso innecesario de antirretrovirales dado el riesgo de inducir resistencia (20). En este estudio, la carga viral se evaluó después de la semana 34 en 53 de las 84 mujeres con parto a término, pero en la mayoría (43 pacientes), se hizo dentro del rango recomendado.

En el $8,4 \%$ de las pacientes con carga viral de 1.000 o más copias $/ \mathrm{ml}$ (20\%), la carga se cuantificó después de las 34 semanas, un porcentaje 
mucho menor que el encontrado en otras series colombianas (58,2\%) (10). En el país todavía una gran parte de la población no accede a la evaluación oportuna de la carga viral, aspecto que debe fortalecerse en el seguimiento de las mujeres gestantes. Si se logra un mayor porcentaje de evaluaciones de la carga viral después de la semana 34, se ayudaría a definir mejor la vía del parto o la administración de la infusión de zidovudina endovenosa en pacientes con cargas virales mayores de 1.000 copias $/ \mathrm{ml}$.

En cuanto al estado de inmunosupresión, en $18,4 \%$ de las 92 pacientes con conteo de linfocitos T CD4+, este fue menor de 200 células por $\mathrm{ml}$, porcentaje similar al encontrado en un estudio en Bogotá (16\%) (10).

El parto fue por cesárea en el $90,6 \%$ de las pacientes de este estudio. El criterio para practicarla fue una carga viral de 1.000 o más copias $/ \mathrm{ml}$ en las semanas 34 a 36, criterio que se cumplió en $8,4 \%$ de las mujeres gestantes y se desconocía en el $42,4 \%$. El resto de las cesáreas se practicaron en cumplimiento del protocolo institucional que, hasta el 2014, establecía que esta intervención debía practicarse en todas las mujeres gestantes con diagnóstico de infección por HIV, dado que la mayoría de infecciones verticales se presentan durante el parto (21).

Además, este porcentaje concuerda con los reportados en otros estudios, de 84 a $95 \%(8,10,22)$. El $91 \%$ de las madres recibieron infusión de zidovudina intravenosa durante el parto, estrategia que ha demostrado reducir la transmisión materno-infantil (23) y que debe emplearse en todos los partos de mujeres con infección por HIV y una carga viral mayor de 1.000 copias/ml; esta cifra se ajusta a las metas locales y es similar a lo encontrado en otras poblaciones del país (92,5\%) (10).

Según la UNICEF, cada año en el mundo nacen, aproximadamente, 20 millones de niños con bajo peso (menos de 2,5 kg), lo que corresponde al $14 \%$ de todos los nacidos vivos. En Colombia, la prevalencia fue de $8,81 \%$ en el 2015 y, en Medellín, del $9 \%$ (24). En este estudio, 26 (25\%) recién nacidos tuvieron un peso de menos de $2.500 \mathrm{~g}$; excepto en dos casos, estos bebés tenían factores asociados con el bajo peso, como el parto prematuro o tener madres que consumían sustancias psicoactivas, eran menores de 18 años o tenían sífilis.

El baño del recién nacido inmediatamente después del nacimiento, se ha planteado como una estrategia adicional para reducir la transmisión del virus (25) y se empleó en el 92,5\% de la población estudiada. El lavado gástrico también se contempla como medida para disminuir la transmisión; no obstante, no es una estrategia ampliamente reconocida y posiblemente se requieran más estudios para justificar su inclusión en los protocolos, por lo que solo se usó en una cuarta parte de los bebés. Por ahora, se desconoce el impacto de estas medidas.

Según los resultados del estudio, los niños tuvieron un acceso adecuado al seguimiento y en el $85 \%$ de ellos se pudo descartar el diagnóstico antes de los 12 meses de edad. Sin embargo, la situación ideal (primera evaluación de carga viral al cabo del primer mes y la segunda a los cuatro meses de vida) (11) no se cumplió, pues en el $40 \%$ de los niños la segunda evaluación de la carga viral se hizo después de los cuatro meses, lo que refleja una falla del sistema de salud. No se registró transmisión materno-infantil en ninguno de los niños que tuvieron dos evaluaciones de carga viral, resultados similares a los hallados en un estudio del 2009 en 23 niños expuestos al HIV materno, los cuales fueron todos negativos para la infección (9). 
La transmisión materno-infantil del HIV ocurre principalmente en el momento del parto; en este estudio se pudo constatar que hubo una adecuada implementación de las estrategias periparto, con un elevado porcentaje de cesáreas, lo cual podría explicar dicho resultado. En estudios en países de Suramérica se han reportado porcentajes de cesáreas entre 1,1 y $4 \%$ $(14,22,26)$. No se pudo establecer la causa de la muerte de los dos pacientes fallecidos, sin embargo, tenían otros factores de riesgo, aunque es de resaltar que la primera carga viral en ambos casos fue negativa al mes del nacimiento.

Es fundamental fomentar estrategias educativas en los programas de pregrado en medicina que aporten elementos clave para prevenir el contagio con el HIV. Se deben promover actividades de salud pública y trabajo comunitario que involucren a los adolescentes y adultos jóvenes, encaminadas a construir proyectos de vida y reforzar la identidad personal, la identidad de género, la planificación familiar, la detección de conductas de riesgo, la prevención de la infección con los diferentes métodos disponibles y la consulta precoz en caso de dificultades y dudas.

Asimismo, el médico general, quien frecuentemente es el primer profesional que tiene contacto con la mujer embarazada, debe tener claridad sobre las estrategias recomendadas para evitar la transmisión maternoinfantil de la infección por HIV.

En los programas de pregrado deberían incluirse actividades formativas específicas sobre la prevención de la infección por HIV en tanto que en los posgrados de pediatría y de ginecología y obstetricia deberían hacerse rotaciones clínicas en infectología perinatal que incluyan seminarios de infección materno-neonatal por el HIV y que permitan conocer su fisiopatología, así como promover las actividades preventivas, diagnósticas, terapéuticas y de seguimiento oportuno de madres e hijos.

Cabe mencionar que una de las limitaciones del estudio es que, durante la recolección de datos, se pudieron haber dejado por fuera a algunos neonatos cuya historia no incluía el código de diagnóstico CIE-10 Z206 ni al ingreso ni al egreso. Para minimizar esto, se consultó igualmente el registro de enfermedades infecciosas que se solicita de rutina para evaluar a los recién nacidos expuestos. Además, debe destacarse la falta de articulación transversal de los programas, lo cual dificultó o impidió el seguimiento de todos los pacientes. Por la forma en que se registra el inicio del tratamiento en las historias clínicas (por lo general, en trimestres, no en semanas), no fue posible establecer la oportunidad del inicio del tratamiento antirretroviral; además, antes del 2014 las guías indicaban iniciarlo después de la semana 14, por lo que no se pudo verificar si había habido retraso a partir del diagnóstico.

En este estudio, los casos de transmisión perinatal en los pacientes en quienes se aplicaron las estrategias durante el parto, no excedieron las metas para el año 2015. No obstante, hay aspectos en la atención prenatal que pueden mejorarse, por ejemplo, hacer la prueba de tamización en el primer trimestre, iniciar tempranamente el control prenatal y el tratamiento antirretroviral, y evaluar la carga viral entre las semanas 34 y 36 , entre otros. Se está cumpliendo con el seguimiento en la mayoría de los recién nacidos hijos de madres con HIV, pero este podría ser más oportuno para confirmar o descartar el diagnóstico tempranamente. En las madres, las infecciones concomitantes más frecuentes fueron la infección por el virus del papiloma humano y la sífilis, condiciones que deben detectarse a tiempo para evitar la morbilidad materno-infantil. 


\section{Agradecimientos}

A la Secretaría de Salud de Medellín y al Hospital Universitario San Vicente Fundación, por el acceso a la información necesaria para el desarrollo del estudio.

\section{Referencias}

1. ONUSIDA. El sida en cifras 2015. Fecha de consulta: 23 de marzo de 2017. Disponible en: http://www. unaids.org/sites/default/files/media asset/AIDS by the numbers 2015 es.pdf

2. UNAIDS. Epidemiological Fact Sheets on HIV/AIDS and sexually transmitted infections. Fecha de consulta: 20 de octubre de 2018. Disponible en: http://data.unaids.org/ publications/fact-sheets01/colombia_en.pdf

3. Mirza A, Rathore MH. Pediatric HIV infection. Adv Pediatr. 2012;59:9-26. https://doi.org/10.1016/j.yapd.2012.04.012

4. World Health Organization. Prevention of mother-to-child transmission. Global Health Observatory data repository. Fecha de consulta: 28 de septiembre de 2016. Disponible en: http://www.who.int/hiv/topics/mtct/en/

5. UNAIDS. Progress Report on the Global Plan. 2015. Fecha de consulta: 16 de abril de 2017. Disponible en: http://www.unaids.org/sites/default/files/media_asset/ JC2774_2015ProgressReport_GlobalPlan_en.pdf

6. Ministerio de Salud y Protección Social. Boletín Epidemiológico. Situación del VIH/ Sida Colombia 2013. Fecha de consulta: 26 de septiembre de 2016. Disponible en: https://www.minsalud.gov.co/Documentos\%20y\%20Publicaciones/BOLETIN\%20 EPIDEMIOLOGICO\%20VIH\%201983-2012.pdf

7. UNAIDS. Informe de brechas en prevención 2016. Fecha de consulta: 11 de marzo de 2017. Disponible en: http://www.unaids.org/sites/default/files/media asset/prevention-gapreport-summary es.pdf

8. García R, Prieto F, Arenas C, Rincón J, Caicedo S, Rey G. Reducción de la transmisión madre hijo del VIH en Colombia: dos años de experiencia nacional, 2003-2005. Biomédica. 2005;25:547-64. https://doi.org/10.7705/biomedica.v25i4.1382

9. Gómez C, Archila ME, Rugeles C, Carrizosa J, Rugeles MT, Cornejo JW. A prospective study of neurodevelopment of uninfected children born to human immunodeficiency virus type 1 positive mothers. Rev Neurol. 2009;48:287-91.

10. Bolívar HS, Gómez-Ríos AC, Carrillo-Rozo ME, Rodríguez-Ortiz JA. Morbilidad materno perinatal en pacientes con infección por VIH, Hospital Simón Bolívar, 2003 - 2011, Bogotá (Colombia). Rev Colomb Ginecol Obstet. 2014;65:308-16.

11. Panel on Treatment of HIV-Infected Pregnant Women and Prevention of Perinatal Transmission. Recommendations for use of antiretroviral drugs in pregnant HIV-1- infected women for maternal health and interventions to reduce perinatal HIV transmission in the United States. Fecha de consulta: 15 de febrero de 2016. Disponible en: https://aidsinfo.nih. gov/contentfiles/lvguidelines/PerinatalGL.pdf

12. Ovalle A, Vizueta E, Casals A, Northland R, González R, Labbe E. Infección por virus de inmunodeficiencia humana en la embarazada. Importancia del conocimiento de la infección en el embarazo y factores de riesgo en la transmisión perinatal. Rev Med Chil. 2003;131:633-40. https://doi.org/10.4067/S0034-98872003000600007

13. Taylor AW, Nesheim SR, Zhang X, Song R, Fitz Harris LF, Lampe MA, et al. Estimated perinatal HIV infection among infants born in the United States, 2002-2013. JAMA Pediatr. 2017;171:435-42. https://doi.org/10.1001/jamapediatrics.2016.5053

14. Valdés RE, Sepúlveda MA, Candia PP, Sepúlveda BC, Lattes AK. VIH/sida: comportamiento epidemiológico de la transmisión vertical en el contexto general de la infección en Chile. Rev Chil Obstet Ginecol. 2011;76:52-7. https://doi.org/10.4067/S0717-75262011000100011

15. Ministerio de Salud y Protección Social. Plan estratégico para la eliminación maternoinfantil del VIH y la sífilis congénita 2011. Fecha de consulta: 13 de noviembre de 2014. Disponible en: https://www.minsalud.gov.co/Documentos\%20y\%20Publicaciones/PLAN\%20 ESTRATEGICO\%20ELIMINACION\%20TRANSMISION\%20MATERNO\%2OINFANTIL\%20 \%20VIH\%20-\%20SIFILIS\%202011.pdf 
16. UNAIDS. Treatment, 2015. Fecha de consulta: 3 de noviembre de 2016. Disponible en: http://www.unaids.org/sites/default/files/media asset/JC2484 treatment-2015 en 1.pdf

17. Ministerio de Salud y Protección Social. Guía para el manejo de VIH/sida basada en la evidencia 2016. Fecha de consulta: 3 de marzo de 2017. Disponible en: https://www. minsalud.gov.co/Documentos\%20y\%20Publicaciones/GUIA\%20PARA\%20EL\%20 MANEJO\%20DE\%20VIH\%20SIDA.pdf

18. ONUSIDA. Informe global. Día mundial del sida 2016. Fecha de consulta: 16 de enero de 2017 . Disponible en: http://files.sld.cu/sida/files/2016/12/UNAIDS_FactSheet_es-noviembre-2.pdf

19. Ministerio de Salud y Protección Social, Fondo de Población de las Naciones UnidasUNFPA, Instituto de Evaluación de Tecnologías en Salud-IETS, Asociación Colombiana de Infectología-ACIN. Guía de práctica clínica basada en la evidencia para la atención de la infección por $\mathrm{VIH} /$ sida en adolescentes (con 13 años o más de edad) y adultos. Fecha de consulta: 15 de febrero de 2017. Disponible en: http://gpc.minsalud.gov.co/gpc sites/ Repositorio/Otros conv/GPC VIH adolescentes/GPC Comple VIHADULTOS web.pdf

20. Antunes F, Zindoga P, Gomes P, Augusto O, Mahumane I, Veloso L, et al. Development of nevirapine resistance in children exposed to the prevention of mother-to-child HIV-1 Transmission programme in Maputo, Mozambique. PLoS One. 2015;10:e0131994. https://doi.org/10.1371/journal.pone.0131994

21. Ghent International Working Group on Mother-to-Child Transmission of HIV. Rates of mother-to-child transmission of HIV-1 in Africa, America, and Europe: Results from 13 perinatal studies. J Acquir immune Defic Syndr Hum Retrovirology. 1995;8:506-10.

22. Velásquez $\mathrm{C}$. Resultados de la aplicación de tres guías nacionales para prevenir la transmisión vertical del VIH en el Instituto Nacional Materno Perinatal. Lima, Perú. Rev Perú Exp Salud Pública. 2011;28:492-6.

23. Kourtis AP, Lee FK, Abrams EJ, Jamieson DJ, Bulterys M. Mother-to-child transmission of HIV-1: Timing and implications for prevention. Lancet Infect Dis. 2006;6:726-32. https://doi.org/10.1016/S1473-3099(06)70629-6

24. Así Vamos en Salud. Prevalencia de bajo peso al nacer - Georreferenciado. Fecha de consulta: 13 de marzo de 2017. Disponible en: https://www.asivamosensalud.org/ indicadores/seguridad-alimentaria-y-nutricional/prevalencia-de-bajo-peso-al-nacergeoreferenciado

25. Organización Panamericana de la Salud, Agencia Española de Cooperación Internacional para el Desarrollo. Guía clínica para la eliminación de la transmisión maternoinfantil del VIH y de la sífilis congénita en América Latina y el Caribe 2010. Fecha de consulta: 3 de octubre de 2014. Disponible en: https://www.paho.org/clap/index.php?option=com

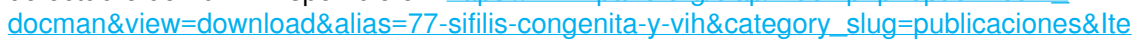
mid $=219 \&$ lang $=$ es

26. Rubio EV, Candia P, Lattes K. Transmisión vertical de VIH y sida: realidad epidemiológica del Cono Sur. Progresos de Obstetricia y Ginecología. 2009;52:511-9. https://doi.org/10.1016/S0304-5013(09)72331-3 